\title{
HOMEOMORPHISM GROUPS OF WEAK SOLENOIDAL SPACES
}

\author{
JAMES T. ROGERS, JR. AND JEFFREY L. TOLLEFSON
}

ABSTRACT. It is shown that inverse limit spaces of closed manifolds with covering maps as bonding maps are determined by their homeomorphism groups.

1. Introduction. A weak solenoidal sequence (solenoidal sequence) of closed manifolds is an inverse limit sequence $(M, f)$ such that each factor space $M_{n}$ is a closed manifold and each bonding map $f_{m}^{n}: M_{n}$ $\rightarrow M_{m}$ is a covering map (regular covering map). The limit space $M_{\infty}$ is called a weak solenoidal space (solenoidal space). If $X$ is a topological space, let $G(X)$ denote the group of homeomorphisms of $X$ onto itself. Give $G(X)$ the point-open topology and consider $G(X)$ as a topological space. The following theorem is our main result.

Theorem. Let $M_{\infty}$ and $N_{\infty}$ be weak solenoidal spaces, and let $\Phi$ be an isomorphism of $G\left(M_{\infty}\right)$ onto $G\left(N_{\infty}\right)$ which is also a homeomorphism. Then $M_{\infty}$ and $N_{\infty}$ are homeomorphic.

A space $X$ is invertible if, for each open set $U$, there is a homeomorphism $h$ of $X$ onto itself that maps $X-U$ into $U$. If $h$ can be chosen to be isotopic to the identity, then we call $X$ continuously invertible. Doyle and Hocking [2] have studied continuous nearhomogeneity as a generalization of continuous invertibility. $X$ is continuously near-homogeneous if given any point $p \in X$ and an open set $U$ of $X$, there is a homeomorphism $h: X \rightarrow X$ such that $h(p) \in U$ and $h$ is isotopic to the identity. We complete this paper by showing that a weak solenoidal space is invertible if and only if it is a solenoid.

If $P=\left(p_{1}, p_{2}, \cdots\right)$ is a sequence of prime numbers (different from $1)$, the $P$-adic solenoid $\Sigma_{P}$ is the limit of the inverse sequence $(M, f)$, where each $M_{n}=\{z:|z|=1\}$ (the unit circle in the complex plane), and each bonding map $f_{n}^{n+1}: M_{n+1} \rightarrow M_{n}$ is defined by $f_{n}^{n+1}(z)=z^{p_{n}}$. The dyadic solenoid $\Sigma_{2}$ is the solenoid corresponding to the sequence $(2,2,2, \cdots)$. The one-dimensional weak solenoidal spaces are precisely the solenoids. D. van Dantzig discussed solenoids in [1].

We follow the notation on inverse limit systems in [4] and restrict

Received by the editors May 23, 1970.

AMS 1969 subject classifications. Primary 5425; Secondary 5480.

Key words and phrases. Homeomorphism groups, inverse limits, weak solenoidal spaces. 
our discussion to inverse limit sequences (the directed set of indices is the positive integers). We assume that all weak solenoidal sequences are nontrivial, i.e., each bonding map is at least a 2-fold covering map. All spaces considered are compact, metric, and nondegenerate and all manifolds are connected. If $x \in X$, the isotropy subgroup at $x$ is the group $G_{x}=\{h \in G(X): h(x)=x\}$. If $X$ is a topological group, then the map $\theta$ of the quotient space $G(X) / G_{x}$ into $X$, given by $\theta\left(g G_{x}\right)=g(x)$, is a continuous bijection. Let $G$ and $H$ be two groups which are also topological spaces (not necessarily topological groups). We say that $G$ is topologically isomorphic to $H$ if there exists an isomorphism of $G$ on to $H$ which is also a homeomorphism.

2. Invertibility and continuous near-homogeneity. The behavior of weak solenoidal spaces with respect to the properties of invertibility and continuous near-homogeneity closely parallels the behavior of closed manifolds. Doyle and Hocking [3] have observed that spheres are the only invertible closed manifolds, and it is easy to see that each closed manifold is continuously homogeneous. We show that solenoids (the only weak solenoidal spaces which are inverse limits of spheres) are the only invertible weak solenoidal spaces, and that each weak solenoidal space is continuously near-homogeneous.

THEOREM 1. Let $M_{\infty}=\lim (M, f)$ be a weak solenoidal space. Then $M_{\infty}$ is continuously near-homogeneous.

Proof. Let $x$ and $y$ be points of $M_{\infty}$, and let $U$ be an open set in $M_{\infty}$ containing $x$. There exists an open cell $C$ in $M_{n}$ (for some $n$ ) such that $x \in f_{n}^{-1}(C) \subset U$. Let $W$ be a closed cell in $M_{n}$ containing $y_{n}$ and $x_{n}$ in its interior. There is a homeomorphism $h_{n}: M_{n} \rightarrow M_{n}$ such that $h_{n}\left(y_{n}\right)=x_{n}$ and $h_{n}$ is the identity on the complement of $W$. Since $W$ is evenly covered by disjoint collections of closed cells, we may lift $h_{n}$ up the inverse sequence to obtain the commutative diagram

$$
\begin{aligned}
M_{n} & \leftarrow M_{n+1} \leftarrow M_{n+2} \leftarrow \cdots \\
h_{n} \downarrow & h_{n+1} \downarrow h_{n+2} \downarrow \\
M_{n} & \leftarrow M_{n+1} \leftarrow M_{n+2} \leftarrow \cdots .
\end{aligned}
$$

Let $h: M_{\infty} \rightarrow M_{\infty}$ be the homeomorphism induced by the sequence of homeomorphisms $\left\{h_{n}\right\}$. Then $(h(y))_{n}=x_{n}$, so $h(y) \in f_{n}^{-1}\left(x_{n}\right)$ $\subset f_{n}^{-1}(C) \subset U$. Since $h$ is isotopic to the identity map, $M_{\infty}$ is continuously near-homogeneous. $\square$

Doyle and Hocking [3], considering the dyadic solenoid as the intersection of solid tori, have shown that it is invertible. This can be done more generally by an inverse limit argument. 
THEOREM 2. If $M_{\infty}$ is a weak solenoidal space, then the following are equivalent:

(a) $M_{\infty}$ is a solenoid;

(b) $M_{\infty}$ is continuously invertible;

(c) $M_{\infty}$ is invertible.

Proof. Suppose $M_{\infty}$ is a solenoid. To prove that $M_{\infty}$ is continuously invertible, it suffices to construct a homeomorphism $h$ of $M_{\infty}$ onto itself that maps the complement of a basic open set $U$ into $U$ such that $h$ is isotopic to the identity. $U$ is of the form $f_{n}^{-1}[(a, b)]$, where $(a, b)$ is an open segment in $M_{n}$.

Let $h_{n}$ be a homeomorphism of $M_{n}$ onto itself of degree 1 which maps the complement of $(a, b)$ into $(a, b)$. Let $J_{n}$ be an isotopy between $h_{n}$ and the identity map. For each $m>n, J_{n}$ lif ts to an isotopy between the identity map of $M_{m}$ and a homeomorphism $h_{m}: M_{m} \rightarrow M_{m}$. Hence $J_{n}$ lifts to an isotopy $J_{\infty}$ between the identity map of $M_{\infty}$ and a homeomorphism $h: M_{\infty} \rightarrow M_{\infty}$ such that $h$ maps the complement of $U$ into $U$.

Suppose that $M_{\infty}$ is invertible. Let $U_{1}$ be an open $n$-cell in $M_{1}$, and let $U=f_{1}^{-1}\left(U_{1}\right)$ be a basic open set in $M_{\infty}$. $U$ is homeomorphic to the product of $U_{1}$ and a Cantor set. For each $n, f_{n}(U)$ is a finite number of disjoint open $n$-cells in $M_{n}$. If $M_{\infty}$ is not a solenoid, then $M_{n}-f_{n}(U)$ is compact and connected. Thus $M_{\infty}-U$ is connected and compact. Let $h$ be a homeomorphism of $M_{\infty}$ onto itself which maps $M_{\infty}-U$ into $U$. Then $h\left(M_{\infty}-U\right)$ is a subset of an $n$-cell. This contradiction completes the proof.

3. Homeomorphism groups. In this section we show that the homeomorphism groups of weak solenoidal spaces determine the spaces.

First we quote a theorem of E. S. Thomas, Jr. [8].

Lemma 1. Suppose that $X_{1}$ and $X_{2}$ are spaces, and suppose that $G\left(X_{1}\right)$ and $G\left(X_{2}\right)$ are topologically isomorphic. Then sufficient conditions that $X_{1}$ and $X_{2}$ be homeomorphic are (for $\left.i=1,2\right)$ :

Condition A. Let $P$ and $Q$ be finite disjoint sets, let $y$ be a point of $X_{i}-P$, and let $V$ be an open set in $X_{i}$. If some member of $G\left(X_{i}\right)$ maps $y$ into $V$, then some member of $G\left(X_{i}\right)$ maps $y$ into $V-Q$, leaving $P$ pointwise fixed.

Condition B. If $\left\{x_{n}\right\}$ is a sequence not converging to $x \in X_{i}$, then there is a subsequence $\left\{y_{n}\right\}$ of $\left\{x_{n}\right\}$ and a map $g$ in $G\left(X_{i}\right)$ such that $g\left(y_{n}\right)=y_{n}$ for all $n$ and $g(x) \neq x$. 
LEMмA 2. If $M_{\infty}$ is a weak solenoidal space which is not a solenoid, then $M_{\infty}$ satisfies both Condition A and Condition B.

Proof. We notice that since $M_{\infty}$ has dimension $>1, W$ in the proof of Theorem 1 can be chosen so that $f_{n}^{-1}(W) \cap P=\varnothing$. Hence the constructed homeomorphism $h$ leaves $P$ pointwise fixed. Furthermore, we can choose the point $x$ so that $Q$ does not intersect $f_{n}^{-1}\left(x_{n}\right)$. Hence $M_{\infty}$ satisfies Condition A.

Let $\left\{x_{n}\right\}$ be a sequence in $M_{\infty}$ not converging to $x$. Let $\left\{y_{n}\right\}$ be a subsequence of $\left\{x_{n}\right\}$ which converges to a point $y$ in $M_{\infty}, y \neq x$, such that $y_{n} \neq x$, for all $n$. Let $U$ be an open set in $M_{\infty}$ which contains $x$ and does not contain any $y_{n}$. Let $C$ be an open cell in $M_{k}$ such that $x \in f_{k}^{-1}(C) \subset U$. Let $h_{k}$ be a homeomorphism of $M_{k}$ onto itself such that $h_{k}\left(f_{k}(x)\right) \neq f_{k}(x)$ and $h_{k}$ is the identity outside $U$. For each $m>k, h_{k}$ lifts to a homeomorphism $h_{m}: M_{m} \rightarrow M_{m}$. The sequence $\left\{h_{m}\right\}$ induces a homeomorphism $h$ of $M_{\infty}$ onto itself such that $h(x) \neq x$ and $h\left(y_{n}\right)$ $=y_{n}$, for all $n$. Therefore, $M_{\infty}$ satisfies Condition B. $\square$

REMARK. Solenoids satisfy Condition B but not Condition A.

REMARK. We have actually shown the stronger result that the group of induced homeomorphisms of a weak solenoidal space of dimension $>1$ is a determining subgroup (see [8] for the definition of determining group and [7] for a proof that the induced homeomorphisms do form a group).

LEMмA 3. Let $S_{1}$ and $S_{2}$ be solenoids. If $G\left(S_{1}\right)$ is topologically isomorphic to $G\left(S_{2}\right)$, then $S_{1}$ is homeomorphic to $S_{2}$.

Proof. We can embed $S_{1}$ (as a topological group) in $G\left(S_{1}\right)$ as the group of translations. Hence $S_{1}$ can be embedded as a topological group in $G\left(S_{2}\right)$. Let $\gamma: S_{1} \rightarrow G\left(S_{2}\right)$ denote this embedding.

Let $x$ be a point in $S_{2}$, and let $p: G\left(S_{2}\right) \rightarrow G\left(S_{2}\right) / G_{x}$ be the projection map. Only the identity of $S_{1}$ fixes $x$, so $\theta \circ p \circ \gamma$ is an injective map of the compact space $S_{1}$ into $S_{2}$ (recall $\theta: G\left(S_{2}\right) / G_{x} \rightarrow S_{2}$ ). Hence $S_{1}$ is topologically contained in $S_{2}$. Therefore, $S_{1}$ is homeomorphic to $S_{2}$, for a solenoid contains no proper indecomposable con tinua.

The next lemma is Theorem 3.1 of [8] with a weakening of the hypotheses and a corresponding weakening of the conclusion.

LEMMA 4. Let $F^{\prime}$ and $F$ be spaces, and let $\Phi$ be a topological isomorphism of $G\left(F^{\prime}\right)$ onto $G(F)$. If $F$ satisfies Condition A, then, for each $x$ in $F^{\prime}, \Phi\left(G_{x}\right)$ is the isotropy subgroup $G_{y}$ of some point $y$ of $F$.

Lemma 5. Let $S$ be a solenoid, and let $M_{\infty}$ be a weak solenoidal space. 
If $G(S)$ is topologically isomorphic to $G\left(M_{\infty}\right)$, then $S$ is homeomorphic to $M_{\infty}$.

Proof. If $M_{\infty}$ is a solenoid, then the conclusion follows from Lemma 3. If $M_{\infty}$ is not a solenoid, then $M_{\infty}$ satisfies Condition A. Let $\Phi$ be a topological isomorphism of $G(S)$ onto $G\left(M_{\infty}\right)$. Let $x \in S$; then $\Phi\left(G_{x}\right)=G_{y}$, for some $y$ in $M_{\infty}$.

Let $U$ be a basic open set in $M_{\infty}$ containing $y . \mathrm{Cl}(U)$ is homeomorphic to the product of a Cantor set $A$ with a closed cell $B$. There exists a simple triod $T$ of homeomorphisms of $A \times B$ into itself such that $T \cap G_{y}=\{1\}$ and such that each homeomorphism agrees with the identity on $A \times \partial B$ (since such triods clearly exist for $B$ ). Extend $T$ to all of $M_{\infty}$ by letting each homeomorphism of $T$ be fixed on the complement of $A \times B$.

Thus there exists a triod $\Phi^{-1}(T)$ in $G\left(S_{1}\right)$. Furthermore $\Phi^{-1}(T) \cap G_{x}$ is the identity map. Hence, if $p: G(S) \rightarrow G(S) / G_{x}$ is the projection map, then $\theta \circ p\left(\Phi^{-1}(T)\right)$ is a triod. This is a contradiction, for each solenoid is atriodic.

Finally, combining Lemmas 1, 2, and 5, we obtain the promised result.

TheOREM 3. Let $M_{\infty}$ and $N_{\infty}$ be weak solenoidal spaces. If $G\left(M_{\infty}\right)$ and $G\left(N_{\infty}\right)$ are topologically isomorphic, then $M_{\infty}$ and $N_{\infty}$ are homeomorphic.

\section{BIBLIOGRAPHY}

1. D. van Dantzig, Über topologisch homogene Kontinua, Fund. Math. 15 (1930), 102-125.

2. P. H. Doyle and J. G. Hocking, Continuously invertible spaces, Pacific J. Math. 12(1962), 499-503.

3. - Invertible spaces, Amer. Math. Monthly 68(1961), 959-965. MR 24 \#A1711.

4. S. Eilenberg and N. Steenrod, Foundations of algebraic topology, Princeton Univ. Press, Princeton, N.J., 1952, MR 14, 398.

5. M. C. McCord, Inverse limit sequences with covering maps, Trans. Amer. Math. Soc. 114 (1965), 197-209. MR $30 \# 3450$.

6. J. T. Rogers, Jr. and J. L. Tollefson, Involutions on solenoidal spaces, Fund. Math. (to appear).

7. - Homeomorphisms homotopic to induced homeomorphisms of weak solenoidal spaces (submitted).

8. E. S. Thomas, Jr., Spaces determined by their homeomorphism groups, Trans. Amer. Math. Soc. 126(1967), 244-250. MR 34 \#5068.

Tulane University, New Orleans, Louisiana 70118 